\title{
Equity-based free channels assignment for secondary users in a cognitive radio network
}

\author{
Said Lakhal ${ }^{1}$, Zouhair Guennoun ${ }^{2}$ \\ ${ }^{1}$ Mohammadia School of Engineering, Mohammed 5 University in Rabat, Morocco \\ ${ }^{2}$ ERSC formerly known as LEC, research Center E3S, Morocco
}

\begin{tabular}{l}
\hline Article Info \\
\hline Article history: \\
Received May 31, 2018 \\
Revised Nov 15, 2018 \\
Accepted Nov 23, 2018
\end{tabular}

Keywords:

Cognitive radio network

Jain's equity index

Scheduler

Channels allocation

\begin{abstract}
The present paper addresses the equity issue among the secondary users in a cognitive radio network. After using a multi scheduler algorithm and a fairness metric namely Jain's Equity Index; we enhance the equity between the secondary users' transfer rates by 0.64 in average, relative to a previous work.
\end{abstract}

Copyright (C) 2019 Institute of Advanced Engineering and Science. All rights reserved.

\section{Corresponding Author:}

Said Lakhal,

Mohammadia School of Engineering, Mohammed 5 University in Rabat,

ERSC formerly known as LEC, research Center E3S,

Mohammadia School of Engineering, Ibn Sina Avenue, B.P 765, Agdal, Rabat, Morocco.

Email: said.lakhal.rech@gmail.com

\section{INTRODUCTION}

In a cognitive radio network (CRN) [1], the primary users (PUs) have priority to use the spectra, without privatization; while the secondary users (SUs), wait the cessation of activities of some PUs, for transmitting data. The design of CRN executes four functions, by using one of four techniques, in the aims to reach at some objectives. These functions are called in the following order [2, 3, 4]: spectrum sensing, spectrum management, spectrum mobility and spectrum sharing [5, 6, 7]. The Auctions, Game theory, Markovian queuing model and Multi-agent systems (MAS) represent the famous used techniques for modeling a CRN [8,9], all to achieve some objectives, characterizing the performance of a CRN. These aims are expressed in terms of some quantities to maximize $[10,11,12]$, such as: the equity, transfer rate and the spectrum use. These requirements are also expressed in terms of other quantities to minimize, like: the energy consumption [13], to extend the lifetime of electrical devices; the interference $[14,15]$, to enhance the quality and quantity of transmission [8,9]. The Figure 1 illustrates altogether the CRN functions, techniques, and objectives.

The SUs' packet transmission phase begins as soon as some channels are released by the PUs. Each of these SUs seeks to be the first to transmit. As a result, conflicts are created and the network can be blocked, as well as no licensed user will be able to send their data. In front of this situation, the installation of a scheduler becomes essential, to manage the channel allocation for different SUs, based on performance criteria whose the equity represents an important component.

This scheduler must take into consideration the way of packet transmission and the properties of the used equity metric. The way is explicit by the distribution of SUs on the free channels according to the time slots, and the properties of the metric are four: population and Scale independence, boundedness as well as Continuity. The authors of $[6,13]$ addressed the equity issues, based on a metric measurement. In the two works, the above properties are not mentioned as well as the way of the packet forwarding was not clarified. In [5], the authors studied the equity by using the JEI as a metric, which satisfied the four properties, but 
they did not make a comparison with another work also they treated the groups of SUs rather than the SUs themselves. In [7, 16], the authors used one channel transmission. In [7], the standard deviation is adopted as metric, which not satisfies the above four properties. In [16], the single channel transmission decreases the equity, despite the consideration of the JEI as a metric. In our present work, we take advantage of the benefits of these works, and we improve them to remove their limitations. Explicitly, on one hand, we implement the multi scheduler algorithm in a multi channel network, to produce the scheduling chain. On the other hand, we use JEI as a metric, since it satisfies the four properties. As a result, we improve equity by an average of 0.64 for previous work.

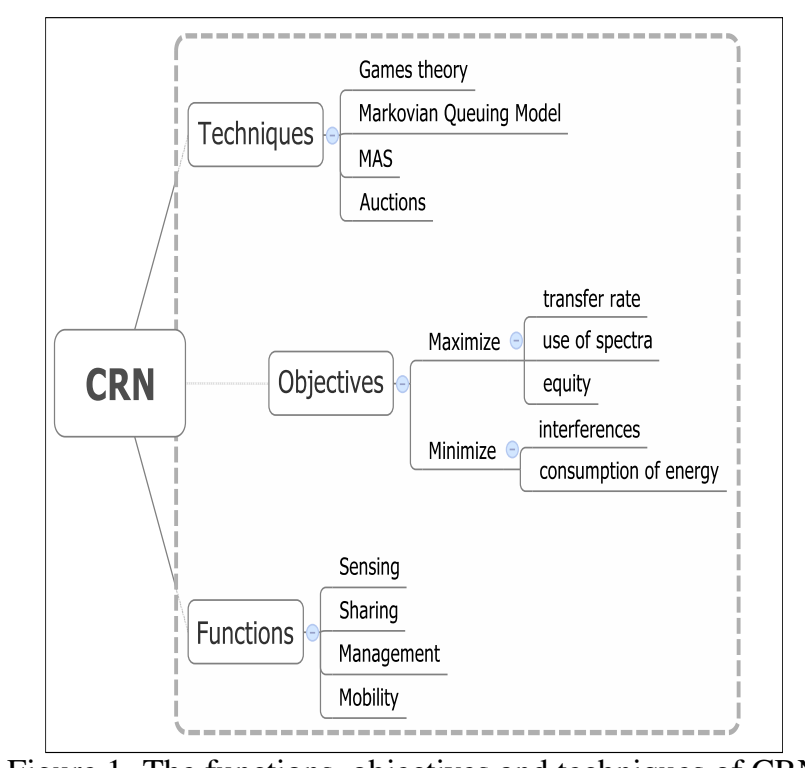

Figure 1. The functions, objectives and techniques of CRN

The rest of this paper is structured as follows: Section 2 discusses some related works and our contributions. Section 3 exposes our approach. We present the experimental results in section 4 , and we conclude in section 5 .

\section{SCHEDULING AND EQUITY CONCEPT: A LITERATURE REVIEW}

\subsection{Scheduling}

The SUs and their packets appear randomly on the CRN. They require the installation of a scheduler, able to managing this flow. This scheduler can be implemented in software or hardware platform. It targets multiple purposes, depending on the priorities and constraints. For example: the priorities can be expressed by the maximization of throughput or equity, while the constraints can be represented by the network infrastructure and architecture. Several previous works treated the throughput maximization problem [11, 17]. Based on constant input and output rates of queues [11], the authors exposed an opportunistic scheduler to enhance the SUs' transmission rates. By referring to the first-difference filter clustering and correlation based PU modeling [17], the authors exposed a QoS-based spectrum coordinator for the CR user cohabitation in order to achieve high throughput and fairness. In addition, the equity criterion between the SUs' transfer rates attracted much attention of researchers $[13,18,5,7]$. So, by taking into account: the current and prior history of user experience, as well as the respect of QoS [13], the authors proposed a fairness approach, by using the JEI. Their aims are to determine the optimal power and rate distribution choices for each SU, while maintaining the fairness across all SUs. In other equity study [18], the authors presented a resource allocation algorithm, in order to maximize the fairness. They used a proportional fairness constraint, to assure that each SU can achieve a required data rate, with QOS guarantees.

Certainly, the adopted architecture affects the network performance. In this regard, we can distinguish between two major architectures: distributed [19, 20, 21] and centralized [22, 7, 5, 19]. Both architectures are considered in [19]. The authors proposed an incentive-aware spectrum sharing scheme, for taking in 
the advantages of these two architectures, and overcoming their weaknesses. The distributed aspect requires no central control entities, which can independently be implemented within a distributed spectrum market. By cons, the centralized aspect assigns the socially optimal contracts for all PUs and SUs, for attaining the economic robustness. In [20], the authors proposed a distributed clustering algorithm based on the soft-constraint affinity propagation message passing model (DCSCAP). So, without depending on predefined common control channel (CCC), DCSCAP relies on the distributed message passing among the SUs, through their available channels, for applying the algorithm on large scale of networks. In a centralized architecture, the authors of [22] proposed a hybrid spectrum sensing method, after creating one of the most trusted spectrums sensing mechanism. The suggested model showed an improvement after comparison with various spectrum sensing methodologies.

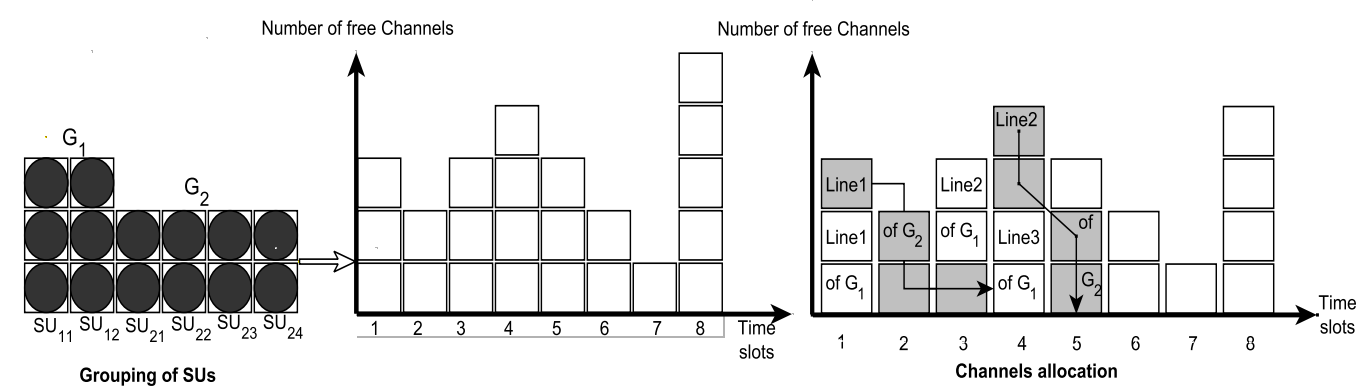

Figure 2. The operating of the mono-scheduler and multi-scheduler algorithms

\subsection{Principles of the mono and multi scheduler algorithms}

In [7], the authors implemented a first algorithm, namely mono-scheduler, to assign the only free channel to different SUs. This algorithm produced a Scheduling String (SS) and used the standard deviation as a metric for evaluating the fairness among the groups of SUs. Its complexity is of order 2, in terms of the total number of packets. The same authors proposed in [5] another algorithm, as an extension of the first, namely multi-scheduler, with a complexity of order 3. This second version improved the mono-scheduler in terms of the number of the free used channels. In the same paper, they used the SS, the States matrix noted $\mathbf{S}$, and the JEI to affect the free channels to the SUs, based on the equity measure, between the groups of SUs. Both [5, 7], considered the fairness among the groups of SUs, but not between the SUs themselves.

Because of the importance of these two algorithms, i.e. mono-scheduler and multi-scheduler, in the present work; we propose to explain the operating principles. To do this, we consider an example of CRN containing:

(i) Two groups of SUs: $G_{1}$ and $G_{2}$, such as: $G_{1}$ has two SUs: $S U_{11}$ and $S U_{12}$, each one has three packets; while $G_{2}$ has four SUs: $S U_{21}, S U_{22}, S U_{23}$ and $S U_{24}$; with two packets for each one as shown in Figure 2;

(ii) Eight for both: number of PUs and that of channels;

After the application of the mono-scheduler algorithm, we obtained: $\mathbf{S S}=\left(G_{1}, 1\right)\left(G_{2}, 1\right)\left(G_{1}, 2\right)\left(G_{2}, 1\right)$. The first component $\left(G_{1}, 1\right)$ of SS signifies that $G_{1}$ transmits one line of packets. This line contains two packets, because $G_{1}$ has two SUs. The second component $\left(G_{2}, 1\right)$ indicates that after the transmission of the first line of $G_{1}, G_{2}$ transmits one line, which contains four packets, since $G_{2}$ has four SUs, and so on. On the other hand, the application of the multi-scheduler algorithm took two arguments: SS and S. This last indicates the state of each channel, during each time slot. To simplify the study, we considered that, the channels have the same capacity. In this case, we were interested only, in the number of the free channels during each time slot, while all channels operated in the same manner. Therefore, $\mathbf{S}$ becomes a row vector, containing in each component, the number of the free channels, during a given time slot. Then, for the example illustrated in Figure 2, we can write: $\mathbf{S}=(3,2,3,4,3,2,1,5)^{T}$. After applying the multi-scheduler algorithm for such example, we could distribute the packets, on the free channels, as shown in Figure 2.

In [16], the authors treated the fairness between the transfer rates of SUs, rather than that between the transfer rates of groups $[5,7]$. They are two Contributions of the present paper: 
(i) Application of the multi-scheduler algorithm [5], for treating the SUs' transfer rates rather than that of groups.

(ii) Enhancement of the SUs' fairness by 0.65 , relative to [16].

\subsection{Equity concept}

In this work, we are interested in calculating the equity between the transfer rates of the SUs, after the application of a scheduler algorithm. In the literature, several works have treated the fairness computation, i.e. fairness metric, among the components of a vector. These works have also studied the properties of this metric. Let $\mathbf{x}=\left(x_{1}, ., x_{i}, ., x_{n}\right)^{T}$ be a vector in $\mathbb{R}_{+}^{n}$, with homogeneous components in terms of measurement unit. In [23], the authors distinguished between four properties of the fairness metric: P1, P2, P3 and P4.

P1: Population size independence: The index should be applicable to any number of components of $\mathbf{x}$, finite or infinite.

P2: Scale and metric independence: The metric should be independent of the scale of all components.

P3: Boundedness: The index should be bounded by two finite values.

P4: Continuity: Any slight change in this metric value, should show a change of at least one component value. They proposed a new fairness metric namely JEI, which verifies together these four properties:

$$
J E I(\mathbf{x})=\left[\sum_{i=1}^{n} x_{i}\right]^{2} \times\left[n \sum_{i=1}^{n} x_{i}^{2}\right]^{-1} .
$$

A value of $J E I(\mathbf{x})$ close to 1 expresses that most of $\mathbf{x}$ components are close to their average. By cons, if they are dispersed around their average, $J E I(\mathbf{x})$ will be close to 0 .

\section{MODELING THE EQUITY}

They are many parameters, which define the modeling of equity. Before beginning this formulation, we prefer to expose these parameters in Table 2.

Table 1. Symbols and Their Meanings

\begin{tabular}{ll}
\hline Symbols & Meanings \\
\hline $\mathrm{m}, \mathrm{n}$ & Number of PUs or channels, and number of current SUs, resp. \\
MaxSUs, $q_{j}$, MaxPack & Max number of SUs, size queue of $S U_{j}$, Max number of packets in a queue, resp. \\
$\mathbf{P}, \mathbf{Q}, e$ & Vector containing the size queue of each $S U$, vector defined based on $\mathbf{P}$, size of $\mathbf{Q}$, resp. \\
$g_{j}, \mathbf{G}, S_{i f}$ & Number of SUs in group $G_{j}$, vector of all $g_{j}$, state of channel i during time slot f, resp. \\
$u_{f}, \mathbf{u}, \mathbf{s}$ & Number of free channels during time slot f, vector of all values of $u_{f}$, size of $\mathbf{u}$, resp. \\
$Q G_{i}, t_{i}, r_{i}$ & Number of packets of $G_{i}$, time for sending all packets of $G_{i}$, transfer rate of $G_{i}$, resp. \\
$t_{i j}, r_{i j}^{*}$ & Time spent to transmit all packets of $S U_{i j}$, transfer rate of $S U_{i j}$, resp. \\
\hline
\end{tabular}

$$
\mathbf{P}=\left(q_{1}, ., q_{j}, ., q_{n}\right)^{T}
$$

We note that during a time fraction, a SU may send one packet or not. We organize the SUs by groups [7], according to the number of packets; such as, a given group, contains all SUs who have the same number of packets. To do this, we define a new vector $\mathbf{Q}$ based on vector $\mathbf{P}$ :

$$
\mathbf{Q}=\left(q_{1}, ., q_{j}, ., q_{e}\right)^{T}, q_{i} \neq q_{j}, \forall(i \neq j, j) \in\{1, ., e\}^{2},(e \leq n), \quad \mathbf{G}=\left(g_{1}, ., g_{j}, ., g_{e}\right)^{T}
$$

We have $m$ channels, and during each time slot, we can find a certain number of free channels. Like that, the state of the CRN is defined by the states of all channels. These states are organized in the matrix $\mathbf{S}$ :

$$
S_{i f}=\left\{\begin{array}{l}
1 \quad \begin{array}{r}
\text { if channel i is free } \\
\text { during time slot } \mathrm{f}
\end{array} \\
0 \quad \begin{array}{l}
\text { if not. }
\end{array}
\end{array}\right.
$$

The number of free channels during a time slot is stored in vector:

$\mathbf{u}=\left(u_{1}, ., u_{f}, ., u_{s}\right)^{T}$ 
During the time slot $\mathrm{f}$, we have $u_{f}$ free channels:

$$
u_{f}=\sum_{i=1}^{m} S_{i f}, f \in\{1, ., s\}
$$

The total number of packets of the group $G_{i}$ in noted $Q G_{i}$. It is obtained by summing the number of packets of all

SUs form group $G_{i}$. By using (2), we can write:

To calculate the transfer rates of groups and those of SUs, we must know the necessary number of passing time slots, for sending all packets.

Let $t_{i}$ and $r_{i}$ be the necessary number of passing time slots for sending all packets and the transfer rate of $G_{i}$, resp.

$$
r_{i}=Q G_{i} \times t_{i}^{-1}=\left(q_{i} \times g_{i}\right) \times t_{i}^{-1}
$$

Let $S U_{i j}$ be the $j^{\text {th }} \mathrm{SU}$ of $G_{i}$. We consider that $S U_{i j}$ needs $t_{i j}$ time slots, for sending all his packets. So, the transfer rate $r_{i j}^{*}$ of $S U_{i j}$, will be given by:

$$
r_{i j}^{*}=q_{i} \times t_{i j}^{-1}
$$

Since $S U_{i j}$ is a member of $G_{i}$. Then, this last completes the transmission of his packets, only after the transmission of all packets of his SUs. Thus, we have the following inequality:

$$
t_{i}-\left(g_{i}-1\right) \leq t_{i j} \leq t_{i}, \quad \forall j \in\left\{1, ., g_{i}\right\}
$$

Let $\mathbf{r}$ and $\mathbf{r}^{*}$ be the vectors containing all transfer rates, of all groups and SUs, respectively.

$$
\begin{gathered}
\mathbf{r}=\left(r_{1}, ., r_{i}, ., r_{e}\right)^{T}, \mathbf{r}^{*}=\left(r_{11}^{*}, ., r_{1 g_{1}}^{*} ; ; r_{i 1}^{*}, ., r_{i g_{i}}^{*} ; . ; r_{e 1}^{*}, ., r_{e g_{e}}^{*}\right)^{T} \\
J E I(\mathbf{q})=\left[\sum_{i=1}^{n} q_{i}\right]^{2} \times\left[n \sum_{i=1}^{n} q_{i}^{2}\right]^{-1}, J E I(\mathbf{Q G})=\left[\sum_{i=1}^{e} Q G_{i}\right]^{2} \times\left[e \sum_{i=1}^{e} Q G_{i}^{2}\right]^{-1} \\
J E I(\mathbf{r})=\left[\sum_{i=1}^{e} r_{i}\right]^{2} \times\left[e \sum_{i=1}^{e} r_{i}^{2}\right]^{-1}, \quad J E I\left(\mathbf{r}^{*}\right)=\left[\sum_{i=1}^{e} \sum_{k=1}^{g_{i}} r_{i k}^{*}\right]^{2} \times\left[n \sum_{i=1}^{e} \sum_{k=1}^{g_{i}} r_{i k}^{* 2}\right]^{-1} .
\end{gathered}
$$

\section{EXPERIMENTATIONS}

We consider four scenarios, containing two, three, four and five groups of SUs, respectively. After, we apply the mono and multi-scheduler algorithms. As a result, we deduct the JEI of the users' rates, which are exposed in Figures 3 and 4.

In all the Figures, we can easily notice that the equities obtained by using our model are very important compared to those of the model [16]. On average, this difference reaches 0.64 , which is explained by the parallel transmission of several packets via several channels in our model. As a result, the users' transfer rates are very close and the equities are very important. By cons, the model of [16] used a single transmission channel, therefore, the standard deviation of the users' transfer rates is very important; then, the equities are very low.
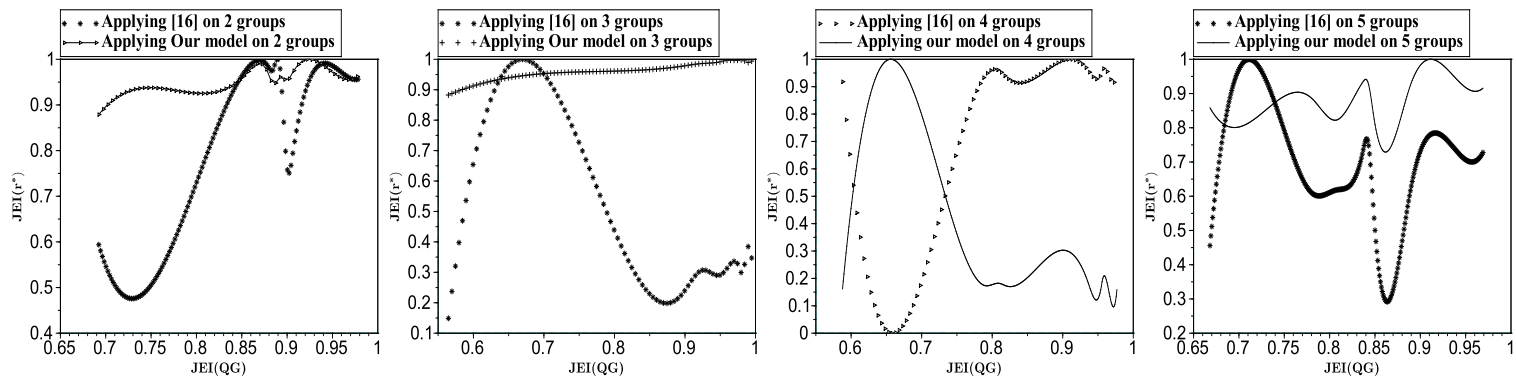

Figure 3. Groups' equities, obtained by using the two models 

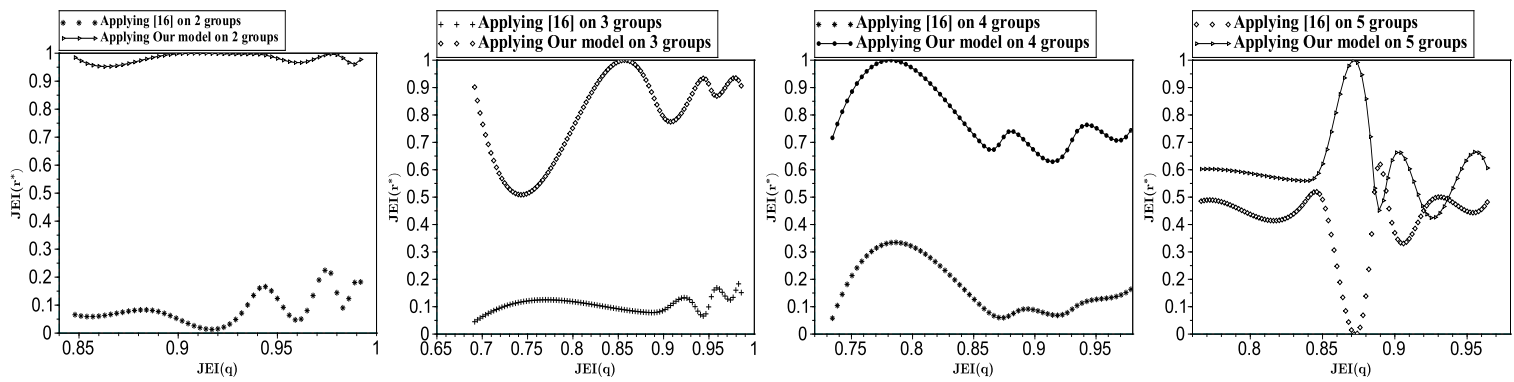

Figure 4. SUs' equities, obtained by using the two models

\section{CONCLUSION}

In this work, we have improved the equity of a previous work, by using a new model. The performance improvement can be explained by the increase of the number of used channels processed by the multi scheduler algorithm. Without forgetting the importance of the monoscheduler, which resides in the establishment of the scheduling chain.

\section{REFERENCES}

[1] Mitola J., "Cognitive radio: an integrated agent architecture for software defined radio," Ph. D. dissertation, Royal Institute of Technology (KTH), Sweden, pp. 8-13, 2000.

[2] I.Akyildiz et, al., "NeXt generation/ dynamic spectrum access/ cognitive radio wireless networks A survey," Computer Networks, vol. 50, pp. 2127-2159, 2006.

[3] A.Khan et, al., "Research Challenges of Cognitive Radio," IJERT, vol. 1, 2012.

[4] P.Bhattacharya et, al., "Smart Radio Spectrum Management for Cognitive Radio," arXiv:1109.0257 [cs], 2011.

[5] A.Idrissi and S.Lakhal, "Equity between secondary users in a cognitive radio network," Wseas, vol. 13, pp. 90-98, 2014

[6] Y.Z.S.Maharjan et, al., "Distributed spectrum sensing in cognitive radio networks with fairness consideration: Efficiency of correlated equilibrium," Mobile Adhoc and Sensor Systems, pp. 540-549, 2011.

[7] A.Idrissi and S.Lakhal, "An algorithm for fairness between secondary users in cognitive radio network," JATIT, vol. 8, 2013.

[8] A.Garhwal and P.Bhattacharya, "A suervey on dynamic spectrum acces techniques for cognitive radio,"arXiv preprint arXiv, pp. 1201-1964, 2012.

[9] B.Benmammar et, al. "A survey on dynamic spectrum acces techniques in cognitive radio networks,"IJCNIS, vol. 5, pp. 68-79, 2013.

[10] HT.Cheng and W.Zhuang, "Simple channel sensing order in cognitive radio networks,"IEEE JSAC, vol. 29, pp. 676-688, 2011.

[11] R.Urgaonkar and M.Neely, "Opportunistic Scheduling with Reliability Guarantees in Cognitive Radio Networks," IEEE transactions on mobile computing", vol. 8, pp. 766-777, 2009.

[12] S.Li et, al., "Maximizing System Throughput by Cooperative Sensing in Cognitive Radio Networks," IEEE/ACM Transactions on Networking, vol. 22, pp. 1245-1256, 2014.

[13] L.Akter and B.Natarajan, "Modeling Fairness in Resource Allocation for Secondary Users in a Competitive Cognitive Radio Network," Wireless Telecommunications Symposium IEEE, pp. 1-6, 2010.

[14] D.Damodaram and T.Venkateswarlu, "Efficient Hardware Architecture for Cyclostationary Detector,"Bulletin of Electrical Engineering and Informatics, vol.5, no.3, pp. 340-346, 2016.

[15] E.A.S.Kang and R.Vig, "Impact of Next Generation Cognitive Radio Network on the Wireless Green Eco system through Signal and Interference Level based K Coverage Probability,'Indonesian Journal of Electrical Engineering and Informatics (IJEEI), vol. 5, no.1, pp. 69-76, 2017.

[16] S.Lakhal and A.Idrissi., "Queues management of secondary users in a cognitive radio network," in NGNS14, pp. 302-307,2014.

[17] C.Berk et, al., "QoS-Aware User Cohabitation Coordinator in Cognitive Radio Networks," IEEE Globecom, pp. 1356-1361, 2012.

[18] Y.Ma et, al., "A Subcarrier-Pair Based Resource Allocation Scheme Using Proportional Fairness for Co- 
operative OFDM-Based Cognitive Radio Networks,” Sensors, vol. 13, pp. 10306-10332, 2013.

[19] Q.Liang et, al., "A distributed centralized scheme for Short and Long Term Spectrum Sharing with a Random Leader in Cognitive Radio Networks,'IEEE JSAC, vol. 30, pp. 2274-2284, 2012;

[20] F.Yao et, al., "Distributed Clustering in Cognitive Radio Ad Hoc Networks Using Soft-Constraint Affinity Propagation," Radioengineering, 2012.

[21] S.Maharjan et, al., "Distributed Spectrum Sensing in Cognitive Radio Networks with Fairness Consideration, Efficiency of Correlated Equilibrium," In IEEE International Conference on Mobile Ad-Hoc and Sensor Systems, pp. 540-549, 2011:

[22] A.S.Khobragade and R.D.Raut, "Hybrid Spectrum Sensing Method for Cognitive Radio," International Journal of Electrical and Computer Engineering (IJECE), vol.7, no. 5, pp. 2683-269551, 2017.

[23] Jain R et, al., "A Quantitive Measure of Fairness and Discrimination for Resource Allocation in Shared Computer Systems," Eastern Research Laboratory, Digital Equipment Corporation Hudson, vol. 38, 1984.

\section{BIOGRAPHIES OF AUTHORS}
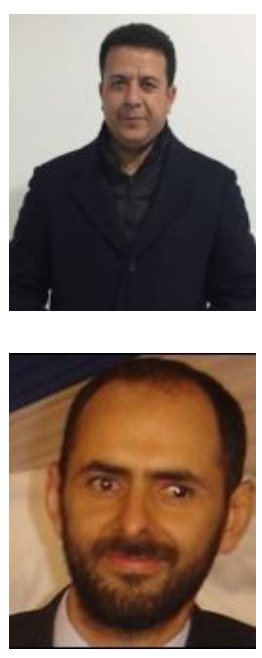

S. Lakhal obtained the diploma of application engineer in computer sciences in 1998, from the University Sidi Mohamed Ben Abdelah, Fes, Morocco, M.Sc. degree in modelization in 2006, from Mohammadia School of Engineering. He is currently a researcher at the Laboratory of Electronics and Telecommunications, Mohammadia School of Engineers (EMI), Rabat, Morocco. His current research interests are Computing, Radio cognitive, Algorithmic and complexity, Modelization.

Z. Guennoun received his engineering degree in Electronics and Telecommunications from the Electronics and Electrical Montefiore Institute, ULG Liege, Belgium in 1987; his M.Sc. degree in Communication Systems from the EMI School of Engineering, Rabat, Morocco in 1993; and his $\mathrm{PhD}$ degree from the same school in 1996. He visited the Centre for Communication Research (CCR) in Bristol University, UK, during the period of 1990-1994 to prepare a split PhD. During 1988-1996 he worked as an Assistant Lecturer in the EMI School of engineering, and from 1996 he is working in the same school as a Professor Lecturer. His fields of interest are digital signal processing, error control coding, speech and image processing. Currently in charge of the laboratory of Electronics and Telecommunications (LEC) at EMI. IEEE member since 1990; and member of the Moroccan IEEE section executive committee. 